\title{
THE SHAPE OF THE BASIN OF ATTRACTION AND TRANSIENTS VIA TRANSFORMATION TO NORMAL FORM
}

\author{
Theo P. VALKERING \\ Center for Theoretical Physics, Twente University of Technology, 7500 AE Enschede, The Netherlands
}

Received 27 February 1986

Revised manuscript received 19 September 1986

\begin{abstract}
A method is derived to approximate the basin and investigate the transients of an attracting fixed point with complex multipliers for an analytic invertible mapping of the real plane. It consists of constructing approximate but explicit expressions for the normal transformation about the fixed point. This transformation has the whole basin as domain, and transforms the nonlinear mapping restricted to the basin, to a linear one.

With this transformation a non-negative functional for the basin is defined whose value decreases along an orbit. Explicit expressions are derived for a sequence of contours approximating a level line of this functional. At large values of the functional a level line approximates the basin boundary.

Calculation of a sequence of level lines yields estimates for that part of the basin where regular (monotonic) convergence to the attracting fixed point is to be expected. The shape of the level lines demonstrates the occurrence of transient-periodic behavior. The way in which the unstable manifold of the saddle, born together with the attractor from a tangent bifurcation, intersects the level lines yields a criterion for a saddle-sink connection. In that case the stable manifold as part of the basin boundary has a simple structure, in contrast to the fractal structure that occurs when there is a homoclinic orbit, which causes final state sensitivity. The calculations are carried out for the Hénon mapping.
\end{abstract}

\section{Introduction}

Consider a dissipative analytic invertible mapping $H, \mathbb{R}^{s} \rightarrow \mathbb{R}^{s}$ with $s \geqq 2$,

$x^{\prime}=H(x), \quad H(x)=P x+Q(x)$,

with an attracting fixed point at the origin. $Q(x)$ contains terms of order two and higher. The linear part $P$ has diagonal normal form with repeated eigenvalues $\lambda_{1}, \ldots, \lambda_{s}$ that satisfy

$\left|\lambda_{1}\right|^{-1}\left|\lambda_{s}\right|^{2} \equiv \delta<1, \quad 0<\left|\lambda_{1}\right| \leqq \cdots \leqq\left|\lambda_{s}\right|<1$.

A sequence of coordinate transformations $u_{n}(x)$ (eq. (3.4)) is derived, that converges with $\delta^{n}$ (theorem 3.2, appendix B) to an invertible real analytic function $\bar{u}(x)$, the normal transformation [1]. This function transforms all orbits of $H$ in the basin of the origin to orbits of the linear map $u^{\prime}=P u$. The transformation $\bar{u}(x)$ contains all nonlinear aspects of the dynamics in the basin. Hence the converging sequence $\left\{u_{n}(x)\right\}$ provides a way to derive qualitative and quantitative results about the basin boundary and about transients in the basin.

In this paper we obtain such results for a mapping of the plane with a fixed point that has two complex eigenvalues $\lambda$ and $\lambda^{*}$. The main tool is the computation of level lines of a functional $\bar{L}(x)$ that is defined on the basin with the aid of $\bar{u}(x)$. The value of this functional decreases monotonically along an orbit in the basin $B_{0}$,

$$
\bar{L}(H x)=|\lambda| \bar{L}(x), \quad x \in B_{0} .
$$

Its level lines are closed nonintersecting contours about the origin (cf. figs. 2-3). Each level line is the locus of points in the basin with equal 'travel 
time' to a given elliptic $\varepsilon$-neighbourhood of the origin. Calculation of a level line at large $L$ yields an estimate of the basin boundary.

Explicit expressions are derived for closed curves $C_{n, L}$, each enclosing an area $\pi L^{2}$ if the Jacobian of the map is constant, that approximate the level line at $\bar{L}(x)=L$ for large $n$.

For any $H(x)$ (cf. (1.1)) one can obtain (and we do this for the Hénon mapping [2])) an expression for a number $n(L)$ such that all points enclosed by $C_{n, L}$ are in the basin if $n>n(L)$. Furthermore the area enclosed by these $C_{n, L}$ is mapped into itself by $H$.

Level lines are calculated for the Hénon mapping. Their shape reflects two properties of the transients. For large $L$ this shape can be very irregular, showing long tentacles (cf. figs. 2-3). This corresponds to strongly nonmonotonic approach of the orbits to the origin. Moreover the area in the plane where this nonmonotonic behavior occurs increases if at fixed Jacobian the other parameters are changed towards the period doubling point of the attractor.

One also sees a second well-known transient phenomenon, namely that an orbit seems to approach an attractor, e.g. a period $q$ orbit, but eventually limits on another one [13]. Corresponding to this phenomenon, the shape of the level lines has a $q$-lobed structure (cf. fig. 3), already before the bifurcation of a period $q$ saddle-node pair.

Conclusions about the structure of the basin boundary (final state sensitivity $[4,5]$ ) are obtained from the way in which the unstable manifold of the saddle accompanying the attracting origin, intersects the level lines. If there are such intersections, the stable manifold is (part of) the basin boundary. Moreover if a sufficiently large part of the unstable manifold $W^{\mathrm{u}}$ of the saddle is enclosed by a level line, one concludes that there is a saddle-sink connection. Then that part of the basin boundary that consists of the stable manifold $W^{s}$ has a simple structure, i.e. there is no final state sensitivity. This contrasts with the situation where $W^{s}$ and $W^{\mathrm{u}}$ intersect transversally. In that case there is final state sensitivity and $W^{\mathrm{s}}$ is part of the boundaries of the basins of infinitely many other attracting orbits as well.

Figs. 2 and 3 show level lines for the Hénon mapping keeping the Jacobian constant and varying the second parameter. More details about the calculations and interpretation of the results are given in section 2. In section 3 the approximations to the normal form transformation and the constructure procedure of the level lines are derived.

\section{Level lines for the Hénon mapping}

Consider the Hénon mapping in the form [6]

$H\left(\begin{array}{l}x_{1} \\ x_{2}\end{array}\right)=P\left(\begin{array}{l}x_{1} \\ x_{2}\end{array}\right)+\left(\begin{array}{c}2 x_{1}^{2} \\ 0\end{array}\right), \quad P=\left(\begin{array}{cc}2 c & -b \\ 1 & 0\end{array}\right)$.

The eigenvalues $\lambda_{ \pm}$of the linear part $P$ are complex if $b>c^{2}$,

$\lambda_{ \pm}=c \pm \mathrm{i}_{\sqrt{ }}\left(b-c^{2}\right), \quad\left|\lambda_{ \pm}\right|=b^{1 / 2}$.

The second fixed point is a saddle at

$x_{1}=x_{2}=a \equiv(b+1-2 c) / 2$,

with multipliers

$\lambda_{u, s}=b+1-c \pm \sqrt{ }\left\{(b+1-c)^{2}-b\right\}$,

both positive. These two fixed points originate from a saddle-node bifurcation at $b=2 c-1$ (cf. fig. 1).

An estimate of the shape of the basin is obtained by calculating level lines $C_{L}$ at large $L$. Since the basin boundary coincides with the "level line" $C_{\infty}$, this estimate can be made arbitrarily accurate. This procedure yields an underestimate: The area enclosed by $C_{L}$ is wholly contained in the basin. To appreciate the usefulness of this estimate note that an overestimate is found by constructing the stable manifold $W^{\mathrm{s}}$ of the saddle. If $P_{0}$ denotes the region enclosed by this manifold 


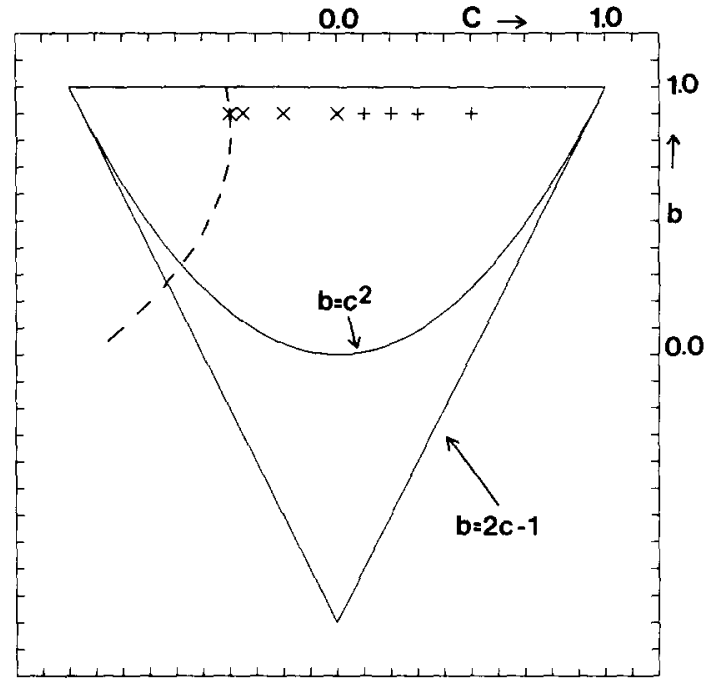

Fig. 1. Parameter diagram for the Hénon mapping (2.1). The attractor at the origin and the saddle are born in a saddle-node bifurcation at $b=2 c-1$. Above the curve $b=c^{2}$ the attractor has complex multipliers. A period-3 saddle-node pair bifurcates at the dashed line (for decreasing $c$ ). Marks + and $X$ indicate the parameter values of the calculations of figs. 2 and 3 , respectively.

containing the origin*, then $B_{0} \subset P_{0}$. Note however that $P_{0}$ may contain the basins of other attractors as well (cf. the period-3 orbit in fig. 3iv)). Examples are the periodic orbits that bifurcate at $b=1$ from the origin, which can be continued in the $b-c$ plane $[7,9,10]$, and those related to the existence of a homoclinic orbit $[3,11]$.

Furthermore the approximation of the basin by $D_{L}$, which denotes the interior of $C_{L}$, is uniform in the following sense: $C_{L}$ is mapped onto $C_{L b^{1 / 2}}$ by $H$. Consequently the 'travel time' of a point $x$ on $C_{L}$ to a small elliptic neighborhood $D_{\varepsilon}$ of the origin is given by

$\tau_{\varepsilon, L} \equiv \ln (L / \varepsilon) / \ln b^{-1}$,
i.e. $H^{n} x \in D_{\varepsilon} \quad$ if $n>\tau_{\varepsilon, L}$.

*More precisely: $P_{0}$ is the set of points that can be connected with the origin through a bounded curve that does not intersect $W^{s}$
Thus all points on $C_{L}$ have equal traveltime and $D_{L}$ consists precisely of those points in the basin with traveltime equal to or less than $\tau_{\varepsilon, L}$.

An explicit expression for an arbitrarily large region wholly within the basin can be obtained from theorem 3.4 (see below). For the Hénon mapping the result is that an area $\pi L^{2}$ enclosed by curves (cf. (3.10b))

$$
\begin{gathered}
C_{n, L}=\left\{x \mid x=H^{-n}\left(b^{n / 2} L\left(a_{1} \cos \phi+a_{2} \sin \phi\right)\right),\right. \\
0 \leqq \phi<2 \pi\}
\end{gathered}
$$

is in the basin if $n>2 \ln (L / l) / \ln \left(b^{-1}\right)$, where the constant $l$ is given by

$$
l=2^{-1}\left(1-b^{1 / 2}\right)\left(b^{1 / 2}-|c|\right)\left(b-c^{2}\right)^{-1 / 2} b^{-1 / 2} .
$$

Here $a_{1}$ and $a_{2}$ are the (orthogonal) eigenvectors of the hermitean matrix $A$ (cf. (3.7)), normalized such that $\left(a_{1}, A a_{1}\right)=\left(a_{2}, A a_{2}\right)=1$.

Figs. 2-3 demonstrate the nonmonotonic approach of orbits to the origin. Keep in mind that for a linear map level lines are ellipses. Here we observe that for larger $L$ long tentacles are formed. Thus the distance of $H^{n}(x)$ to the origin is certainly not a monotonic function of $n$, for orbits starting at large $L$. Furthermore note that the area where this nonmonotonic behavior occurs increases with decreasing $c$ (fig. 2). One also observes that the length of grad $\bar{L}$ can be very large in this area. Consequently two points a small distance apart can have a very different travel time to $D_{\varepsilon}$ [12]. This is another aspect of irregular transient behavior.

In view of the 3- or 4-lobed shape of the level lines in fig. 3 , one expects (at the corresponding parametervalues) that many orbits in het basin seem to converge to a period-3 (or -4 ) orbit, but eventually wander away to the origin. In fig. 3iv) this shape is caused by a period-3 saddle-node pair that bifurcates at $b=0.9, c=-0.3991$ (analytical result, cf. [10]). In iii) however, there is no period-3 orbit. Nevertheless the same shape is 

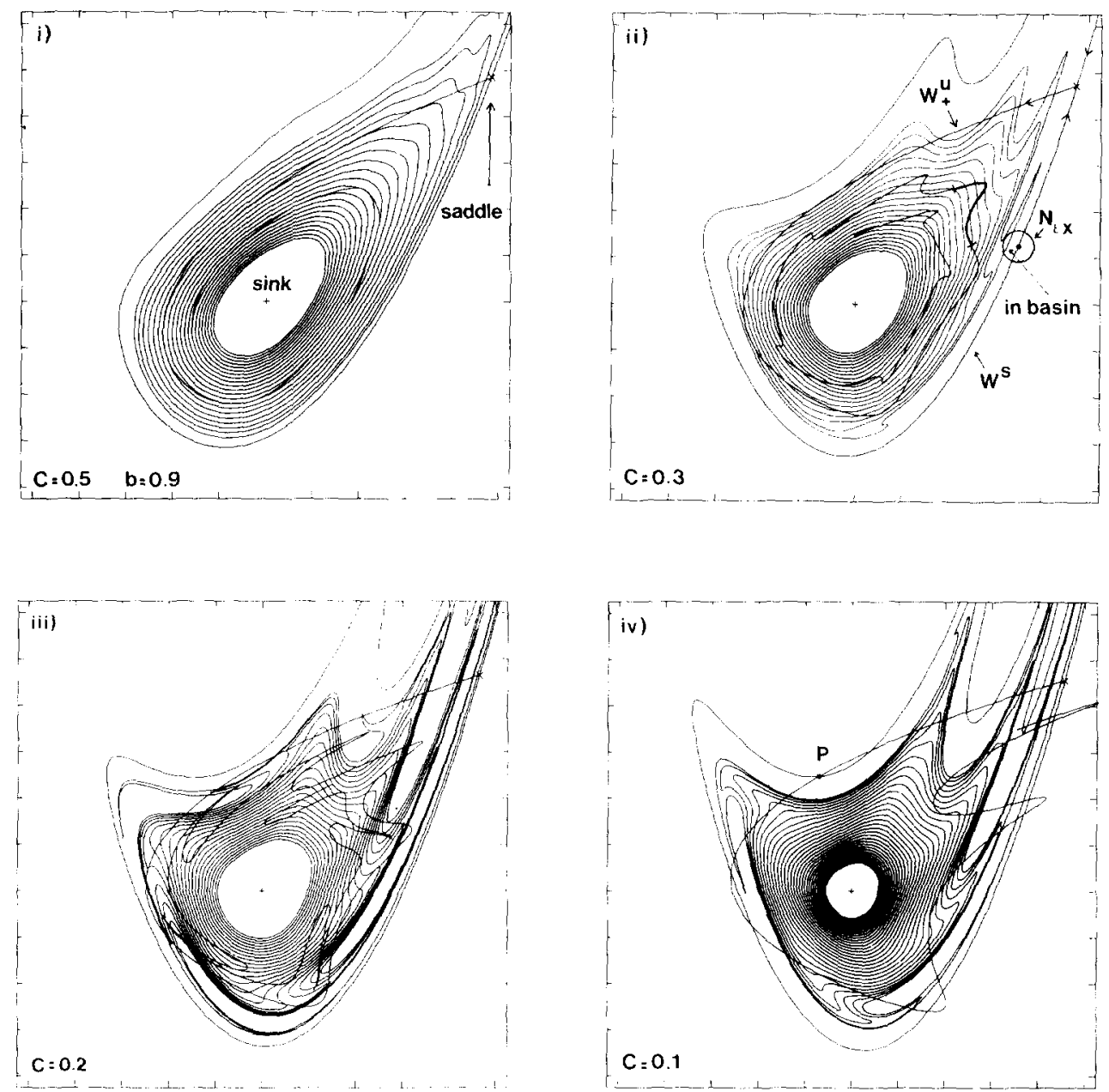

Fig. 2. Level lines of the functional $L(x)(1.2)$ for the Hénon mapping (2.1), evaluated for fixed $b=0.9$ and decreasing $c$. Each level line is the image of the next one outside. The area enclosed by each contour is $0.36 \pi a^{2} b^{n}$ respectively, $n=0,1, \ldots 20$, where $(a, a)$ are the coordinates of the saddle. Figs. i-iv illustrate the decrease of the area when the orbits approach the origin monotonically. The outermost level line provides an estimate of the basin boundary. i, ii): All points of the branch $W^{u}$ of the unstable manifold are in the basin. Hence each $x \in W^{s}$ has a neighborhood $N_{f x}$ of which one connected part is in the basin. The thick part of $W_{+}^{u}$ in ii) denotes a piece $w$, whose images by $H^{j}$ build up $W_{+}^{u}$ completely (see text).

present, due to the existence of two (complex!) period-3 orbits in $\mathbb{C}^{2}$, close to the real plane [13].

From figs. 2-3 one can also infer properties of the basin boundary, especially about final state sensitivity $[4,5]$. In all cases shown, the unstable manifold $W^{u}$ of the saddle intersects a level line. Hence it intersects the basin and consequently the stable manifold is (part of) the basin boundary [8].
In fig. 2i, ii) one observes that all points of the left branch of $W^{u}$ are in the basin, i.e. there is a saddle-sink connection. In that case $W^{\mathrm{s}}$ as part of the basin boundary, has a simple structure: for each $x \in W^{\text {s }}$ there is a neighbourhood $N_{\varepsilon x}$ such that one half (cf. fig. 2ii) is completely in the basin. This is proved in appendix A. There is no final state sensitivity in this case. 

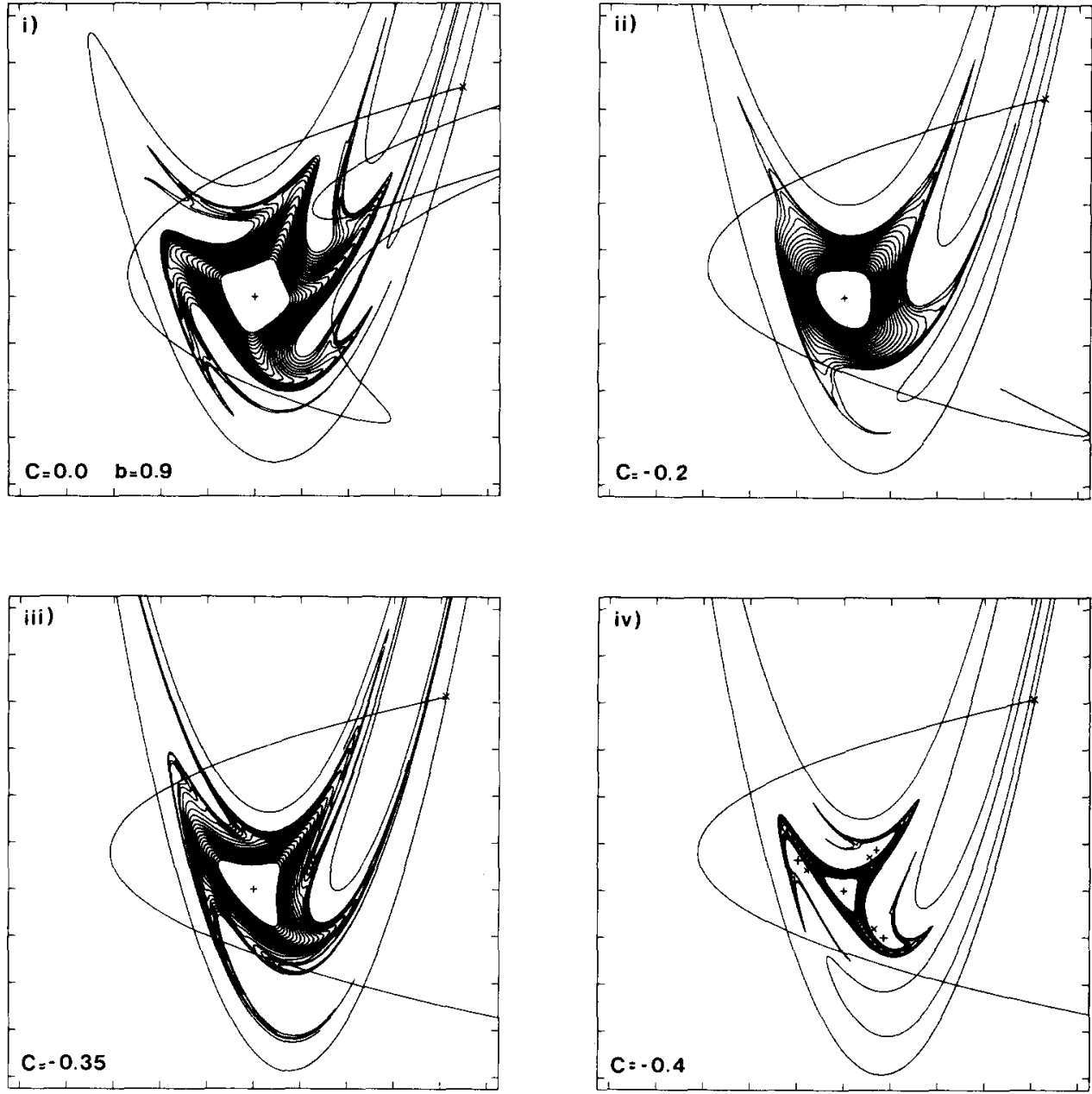

Fig. 3. The $q$-lobed shape $(q=3,4)$ demonstrates the transient approach to a period- $q$ orbit in the basin. In iii) this is caused by the existence of a complex period-3 pair close to the real plane. For smaller values of $c$ this pair bifurcates into a saddle-node pair at $c=-0.3991[10]$. The + and $\times$ in iv) mark the period-3 since and saddle respectively. The enclosed area equals $s \pi a^{2} b^{n}$ with $s$ equal to $0.19,0.16,0.16$ and 0.04 , respectively, and $n=0,1, \ldots, 20$.

In order to establish the saddle-sink connection definitely from in fig. $2 \mathrm{i}$, ii), note that $W^{\mathrm{u}}$ consists of two invariant branches $W_{+}^{\mathrm{u}}$ and $W_{-}^{\mathrm{u}}$ since both multipliers of the saddle are positive. Furthermore, since the map is invertible, one such branch can be written as the union $\bigcup_{-\infty<n<\infty} H^{n} \tilde{w}$, where $\tilde{w}$ is a piece of $W_{+}^{\mathrm{u}}$ between two subsequent point $x_{0} \in W_{+}^{\mathrm{u}}$ and $H x_{0}$. Hence there is a saddle-sink connection if one can find such a piece in the interior of a curve $C_{L}$. This can be read off easily from the intersections of $W_{+}^{u}$ with the level lines. If $W_{+}^{\mathrm{u}}$ intersects a level line $C_{L}$ for the first time at $x_{0}$ (counting from the saddle) then it intersects the next level line for the first time at $H x_{0}$. This determines a piece $\tilde{w}$ (cf. fig. 2ii).

Finally, if there is a homoclinic orbit (cf. figs. 2iv), 3) there certainly is no saddle-sink connection. Nevertheless $W^{s}$ is part of the basin 
boundary. The latter property follows from the fact that one can always find a bounded region (cf. the region bounded by pieces of $W^{\mathrm{s}}$ and $W^{\mathrm{u}}$ between the saddle and the point $p$ in fig. 2iv)) that contains the origin and has parts of $W^{u}$ and $W^{s}$ as boundary. Thus since the area of the basin is infinite for the present (invertible) mapping, this basin necessarily intersects $W^{\mathrm{u}}$, so that $W^{\mathrm{s}}$ consists of boundary points [8].

Extending this argument one can show that $W^{\mathrm{s}}$ belongs to the boundary of each attracting orbit in $P_{0}$. Examples of such orbits are mentioned at the beginning of this section. The presence of a homoclinic orbit causes that $W^{\mathrm{s}}$ piles up against itself. This results in a sensitivity of ' $\varepsilon \ln \varepsilon^{-1}$ ' type with respect to final states either in $P_{0}$ or in its complement [5].

\section{Construction of normal transformation and level lines}

First the existence is established of an analytic transformation $\bar{u}(x)$ from the basin of the origin to $\mathbb{R}^{s}$ which transforms all orbits of the nonlinear map in the basin to orbits of the linear map $u^{\prime}=P u$. Secondly a set of transformations $\left\{u_{n}(x)\right\}$ is derived which approximate $\bar{u}(x)$ for large $n$. Finally the functional $\bar{L}$ (cf. (1.2)) is defined and a procedure to construct its level lines (if $s=2$ ) is formulated. At the same time we obtain explicit expressions for contours about the origin that enclose an arbitrarily large area in the basin.

Following Hartmann's theorem [8], there is a neighbourhood of the origin and a homeomorphism $\hat{u}(x)$ such that an orbit $x_{n}=H^{n}\left(x_{0}\right)$ in this neighbourhood is mapped onto an orbit $u_{n}=P^{n} u_{0}$ of the linear mapping $u^{\prime}=P u$, where $u_{0}=\hat{u}\left(x_{0}\right)$. For maps (1.1) considered here, the inequality (1.1b) guarantees that the eigenvalues of $P$ are nonresonant, and $\hat{u}(x)$ is biholomorphic (Poincaré's theorem [1]). The validity of this local theorem can be extended easily to the whole basin.

Lemma 3.1. There is an analytic transformation $\bar{x}(u)$, with analytic inverse $\bar{u}(x)$, that maps $\mathbb{R}^{s}$ onto the basin of attraction of the origin such that

$H(\bar{x}(u))=\bar{x}(P u), \quad P \bar{u}(x)=\bar{u}(H x)$,

$\bar{x}(u)=u+$ h.o.t.,

and, if $H$ has constant Jacobian,

$\operatorname{det}(D \bar{x}(u))=\operatorname{det}(D \bar{u}(x))=1^{*}$.

Proof. There exists [1] a biholomorphic transformation $\hat{x}(u)=u+$ h.o.t., defined at a small domain about the origin that satisfies $(3.1 \mathrm{a}, \mathrm{b})$. Define $\bar{x}(u)$ for given $u$ outside this domain by

$\bar{x}(u)=H^{-m} \hat{x}\left(P^{m} u\right)$,

where $m$ is such that $P^{m} u$ is in this domain. Obviously $m$ is not unique, but one readily shows that $\bar{x}(u)$ is single-valued (cf. the proof of the lemma in appendix A). This function $\bar{x}(u)$ is analytic, since $\hat{x}(u)$ and $H$ are analytic. With definition (3.2) and the fact that $\hat{x}(u)$ satisfies $(3.1 \mathrm{a}, \mathrm{b})$, one readily verifies $(3.1 \mathrm{a}, \mathrm{b})$ for $\bar{x}(u)$. Similar arguments hold for the inverse $\bar{u}(x)$.

Differentiation of (3.1a) yields, if det $D H(x)$ is constant,

$\operatorname{det}(D \bar{x}(u))=\operatorname{det}(D \bar{x}(P u))$.

Since $\operatorname{det}(D \bar{x}(u))$ is analytic (3.1c) follows immediately with (3.3):

$$
\begin{aligned}
\operatorname{det}(D \bar{x}(u)) & =\lim _{n \rightarrow \infty} \operatorname{det}\left(D \bar{x}\left(P^{n} u\right)\right) \\
& =\operatorname{det}(D \bar{x}(0))=1 .
\end{aligned}
$$

QED

The normal transformation $\bar{x}(u)$ contains all in formation on the nonlinear aspects of the motion. so it is worthwhile to find useful approximations. To this end define the set of transformations

$x_{n}(u)=H^{-n}\left(P^{n} u\right), \quad u_{n}(x)=P^{-n} H^{n}(x)$.

\footnotetext{
${ }^{*} D \bar{x}(u)$ denotes the derivative of $\bar{x}(u)$.
} 
The $x_{n}(u)$ approximate $\bar{x}(u)$ if $\delta$, defined in (1.1b) is less than one.

\section{Theorem 3.2}

i) For each $u \in \mathbb{R}^{s}$ there is a constant $M_{u}$ such that

$\left\|x_{n}(u)-\bar{x}(u)\right\| \leqq M_{u} \delta^{n}$

ii) For each $x$ in the basin $B_{0}$, there is a constant $M_{x}$ such that

$\left\|u_{n}(x)-\bar{u}(x)\right\| \leqq M_{x} \delta^{n}$

Here $\|\cdot\|$ denotes the Euclidean norm (or an equivalent one (cf. (3.11)).

Remark. The proof is given in appendix B. The validity of this theorem is related to the fact that (3.1a) can be written as $\bar{x}(u)=H^{-1} \bar{x}(P u)$, i.e. as a functional equation $\bar{x}(u)=F \bar{x}(u)$ for the function $\bar{x}(u)$. Then $x_{n}(u)$ is the $n$th iterate by $F$ of a zero order approximation $x_{0}(u)=u, x_{n}(u)=$ $F^{n} x_{0}(u)$.

We now apply these general results to a mapping of the plane and to the case that $P$ has complex eigenvalues $\lambda$ and $\lambda^{*}$, and Jacobian $b \equiv$ $\lambda \lambda^{*} ; \delta$ then equals $b^{1 / 2}$. With $\bar{u}(x)$ the Lyapunov functional $\bar{L}(x)$ (cf. (1.2)) will be defined. Let $P$ be diagonalized by a matrix $S$,

$S P S^{-1}=\Lambda, \quad \Lambda \equiv \operatorname{diag}\left(\lambda, \lambda^{*}\right)$.

Then the equalities $\left(P u, S^{\dagger} S P u\right)=\left(u, S^{\dagger} \Lambda^{\dagger} \Lambda S u\right)$ $=|\lambda|^{2}\left(u, S^{\dagger} S u\right)$ yield

$(P u, A P u)=b(u, A u), \quad A=S^{\dagger} S$,

since $|\lambda|^{2}=b$. If a functional $\bar{L}(x)$ is defined

$\bar{L}(x) \equiv(\bar{u}(x), A \bar{u}(x))^{1 / 2}, \quad x \in B_{0}$ then it holds analogous to (3.7)

$\bar{L}(H x)=b^{1 / 2} \bar{L}(x), \quad x \in B_{0}^{\ddagger}$.

Since $A=S^{\dagger} S$ is hermitean with positive eigenvalues and $\bar{u}(x)$ has nonzero Jacobian, $\bar{L}(x)$ is nonnegative and has only one stationary point, a zero at the origin. Consequently, the level lines $C_{L}$

$C_{L} \equiv\left\{x=\bar{x}(u) \mid(u, A u)=L^{2}\right\}$

are closed contours about the origin and they do not intersect. Similarly contours $C_{n, L}$ are defined, which are level lines of the functional $\left(u_{n}(x)\right.$, $\left.A u_{n}(x)\right)$,

$C_{n, L} \equiv\left\{x=x_{n}(u) \mid(u, A u)=L^{2}\right\}$.

From theorem 3.2 one infers immediately:

Corollary 3.3. The contours $C_{n, L}$ converge pointwise to the level line $C_{L}$.

The procedure to approximate a level line simply is to take an ellipse $\left\{u \mid(u, A u)=L^{2}\right\}$ and apply $x_{n}(u)$ for increasing $n$. In this way one obtains the curves $C_{n, L}$. Note that the area enclosed by $C_{n, L}$ equals $\pi L^{2}$ for each $n$ if $H$ has constant Jacobian, since $x_{n}(u)$ is area preserving in that case.

For sufficiently large $n$, depending on $L$, such an area is wholly in the basin. To determine how big $n$ should be it is convenient to introduce an inner product and corresponding norm related to $P$,

$(x, y) \equiv(x, A y), \quad x, y \in \mathbb{R}^{2}$

Henceforth $\|\cdot\|$ denotes the norm derived from the inner product above.

\footnotetext{
\#In the general case of mappings (1.1) $\bar{L}(x)$ can be defined similarly and it holds $\bar{L}(H x) \leqq\left|\lambda_{s}\right| \bar{L}(x)$.
} 


\section{Theorem 3.4}

i) For each mapping (1.1) there is a positive constant $l$ such that the interior of $C_{n, l}$, denoted by $D_{n, L}$ satisfies

$H D_{n, L} \subset D_{n, L} \quad$ if $n>2 \ln (L / l) / \ln \left(b^{-1}\right)$

and

$\lim _{j \rightarrow \infty} H^{j} x=0 \quad$ if $x \in D_{n, L}$.

ii) If the nonlinear term in $Q(x)$ in (1.1) is homogenously quadratic and if $q$ is a constant such that

$\|Q x\| \leqq q\|x\|^{2}$

then $l$ in $(3.12 a)$ can be chosen as

$l=\left(1-b^{1 / 2}\right) / q$.

Proof. Since $Q(x)$ in (1.1) is analytic there are constants $M$ and $R$ such that

$\|Q(x)\| \leqq M\|x\|^{2}, \quad$ if $\|x\| \leqq R$.

Then one obtains with Schwarz' inequality and using the fact that the operator norm of $P$ in the metric derived from (3.11) equals $b^{1 / 2}$ (cf. (3.7))

$$
\begin{array}{r}
\|H x\| \leqq\|P x\|+\|Q(x)\| \leqq\left\{b^{1 / 2}+M\|x\|\right\}\|x\|, \\
\|x\| \leqq R
\end{array}
$$

Let the constant $l$ and the elliptic region $N_{l}$ be defined by

$l \equiv \min \left(R, \frac{1-b^{1 / 2}}{M}\right), \quad N_{l} \equiv\{x \mid\|x\|<l\}$

Then (cf. (3.16)) $\|H x\| \leqq \theta\|x\|$ where $0<\theta<1$ if $x \in N_{\text {, }}$. Consequently

$H N_{l} \subset N_{l}, \quad \lim _{j \rightarrow \infty} H^{j} x=0 \quad$ if $x \in N_{l}$.

The next step is to perform for fixed $m$ a coordinate transformation $y=u_{m}(x)$. The mapping (1.1) then reads

$$
y^{\prime}=H_{m}(y), \quad H_{m}(y)=P y+P^{-m} Q\left(P^{m} y\right) .
$$

Again with (3.7) one concludes easily that (3.15) holds for the nonlinear part of $H_{m}$, with constants $M_{m}$ and $R_{m}$ given by

$M_{m}=b^{m / 2} M \quad$ and $\quad R_{m}=b^{-m / 2} R$.

As a result (3.18) holds for each $x \in N_{l_{m}}$, with $l_{m}=l b^{-m / 2}$. Finally transform back to $x$-space. $N_{l_{m}}$ then transforms to the interior of $C_{m, l_{m}}$ and we obtain $(3.12 \mathrm{a}, \mathrm{b})$ for each pair $\{m, L\}$ such that $L \leqq l_{m}$, which yields the inequality in (3.12a). ii) If $Q(x)$ is homogeneously quadratic, the constant $M$ above equals $q$ (3.13) and the restriction $\|x\| \leqq R$ can be dropped. Eq. (3.14) then follows from (3.17). QED

\section{Acknowledgements}

I thank Robert Helleman for useful discussions. This work was partially supported under DEAC03-84-ER 40182.

\section{Appendix A}

It is proved that the stable manifold of the saddle as part of the basin boundary has a simple structure if there is a saddle-sink connection (cf. fig. 2 ii). First Hartmann's (local) theorem [8] is extended as follows:

Lemma. Let an analytic invertible mapping $T$, $\mathbb{R}^{2} \rightarrow \mathbb{R}^{2}$ have a hyperbolic fixed point $x_{0}$ with characteristic multipliers $\left|\lambda_{u}\right|>1$ and $\left|\lambda_{s}\right|<1$. Let the linearization of $T$ at $x_{0}$. denoted by $D T_{0}$. have eigenspaces $E^{\mathrm{u}}$ and $E^{\mathrm{s}}$ respectively.

Then there is a domain $D$, satisfying

$$
D T_{0} D=D, \quad E^{\mathrm{u}} \subset D, \quad E^{\mathrm{s}} \subset D,
$$


and a continuous transformation $x(u): D \rightarrow \mathbb{R}^{2}$ that maps each orbit of the mapping $u^{\prime}=D T_{0} u$ in $D$ onto an orbit of the mapping $x^{\prime}=T x$, i.e.

$T x(u)=x\left(D T_{0} u\right), \quad u \in D$

Furthermore $x(u)$ is locally a homeomorphism: each $u \in D$ has a neighbourhood on which $x(u)$ has a continuous inverse.

Proof. According to Hartmann's theorem, there is a homeomorphism $\hat{x}(u)$, defined on a neighbourhood $D_{0}$ of $x_{0}$ such that

$$
T \hat{x}=\hat{x}\left(D T_{0} u\right), \quad u \in D_{0}, \quad D T_{0} u \in D_{0} .
$$

For simplicity $D_{0}$ is chosen in such a way that for each $u \in D_{0}$, there exist integers $m_{+}$and $m_{-}, m_{+}$ $\geqq 0, m_{-} \leqq 0$ such that

$D T_{0}^{j} u \in D_{0}, \quad m_{-} \leqq j \leqq m_{+}$,

$D T_{0}^{j} u \notin D_{0}, \quad j>m_{+}, \quad j<m_{-}$.

The domain $D \equiv \bigcup_{-\infty<n<\infty} D T_{0}^{n} D_{0}$ clearly satisfies (A.1). On this domain the function $x(u)$ is defined according to

$x(u)=\hat{x}(u), \quad u \in D_{0}$,

$x(u)=T^{-k} \hat{x}\left(D T_{0}^{k} u\right), \quad u \in D_{0}$,

where $k$ is an integer such that $D T_{0}^{k} u \in D_{0}$. Although for given $u \in D_{0}$ the integer $k$ is not unique, this definition yields a single-valued $x(u)$ : let $k_{1}$ and $k_{2}$ be such that $D T_{0}^{k_{1}} u \in D_{0}, D T_{0}^{k_{2}} u \in$ $D_{0}$. Then $x(u)$ is single-valued iff

$T^{-k_{1}} \hat{x}\left(D T_{0}^{k_{1}} u\right)=T^{-k_{2}} \hat{x}\left(D T_{0}^{k_{2}} u\right)$.

This relation follows immediately from (A.3) and (A.4): let $k_{2}-k_{1}>0$. Then because of (A.4) all points $D T_{0}^{k} u, k=k_{1}, k_{1}+1, \ldots, k_{2}-k_{1}$, are in $D_{0}$. This allows us to apply (A.3) $\left(k_{2}-k_{1}\right)$ times on the left-hand side of (A.6), which yields an identity. $x(u)$ is locally a homeomorphism, since it is a product of homeomorphisms for each $u$ and a neighborhood of $u$ (cf. (A.5)).

Remark. The transformation is generally not invertible on $D$ (for example if there is a homoclinic orbit).

With this lemma we next prove (cf. fig 2ii) and the last paragraphs of section 2)

Theorem. If $W_{+}^{\mathrm{u}}$ is wholly contained in the basin $B_{0}$, then for each $x \in W^{\mathrm{s}}$ there is an $\varepsilon$-neighborhood $N_{\varepsilon x}=\{y\|y-x\|<\varepsilon\}$ that is divided by $W^{s}$ in two connected open parts, such that one part is in the basin.

Proof. Let $E_{+}^{\mathfrak{u}}$ denote that part of $E^{\mathfrak{u}}$ that is mapped by $x(u)$ onto $W_{+}^{\mathrm{u}}$. By assumption each

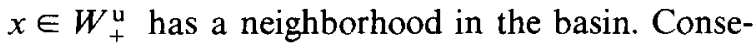
quently each $u \in E_{+}^{u}$ has a neighborhood $\sigma$ such that the image $x(\sigma)$ is in the basin. Each $u \in E^{\mathrm{s}}$ has a neighborhood $\rho$ divided by $E^{s}$ in two open parts $\rho_{+}$and $\rho_{-}$, such that for each $v \in \rho_{+}$there is an $u \in E_{+}^{\mathrm{u}}$, a neighborhood $\sigma$ of $u$ and an $n$ such that $D T_{0}^{n} v \in \sigma$. Consequently $x(v) \in B_{0}$ for each $v \in \rho_{+}$.

Finally, since $x(u)$ is locally invertible, the image of $\rho$ by $x(u)$ is topologically equivalent to $\rho$. This proves the theorem. QED

\section{Appendix B}

In this appendix Theorem 3.2 is proved. For convenience the metric defined in (3.11) is used.

First we prove $(3.5 b)$ in a somewhat stronger version:

For each simply connected bounded domain $D$ in the basin containing the origin there is a constant $C_{D}$ such that

$$
\sup _{x \in D}\left\|u_{n}(x)-\bar{u}(x)\right\| \leqq C_{D} \delta^{n} .
$$

Since the nonlinear part $Q(x)$ of the mapping is 
analytic there is a constant $N_{D}$ such that

$\|Q(x)\| \leqq N_{D}\|x\|^{2}, \quad x \in D$.

Since $D$ is in the basin there is a constant $M_{D}$ such that

$\left\|H^{n} x\right\| \leqq\|P\|_{\mathrm{op}}^{n} M_{D}, \quad x \in D$,

where \|\|$_{\text {op }}$ denotes the operator norm. The difference between two subsequent terms $u_{n}(x)$ reads

$u_{n+1}(x)-u_{n}(x)=P^{-n-1} Q\left(H^{n} x\right)$.

Hence, with (B.2,3) and the fact that in the metric (3.11) the operator norm of $P$ and $P^{-1}$ are $\left|\lambda_{s}\right|$ and $\left|\lambda_{1}\right|^{-1}$ respectively, we obtain a constant $G_{D}$ such that

$$
\begin{gathered}
\sup _{x \in D}\left\|u_{n+1}(x)-u_{n}(x)\right\| \leqq\left|\lambda_{1}\right|^{-(n+1)} . \\
N_{D}\left\|H^{n} x\right\|^{2} \leqq \delta^{n} G_{D} .
\end{gathered}
$$

As a result $u_{n}(x)$ converges to a function $\hat{u}(x)$ in supremum norm. Since $P u_{n}(x)=u_{n-1}(H x)$, this function satisfies $P \hat{u}(x)=\hat{u}(H x)$ and it has to be identified (cf. (3.1a)) with $u(x)$ in (3.5b).

In order to prove (3.5a) define the images of $D$ by $u_{n}(x)$ and $\bar{u}(x)$ respectively

$U_{n} \equiv u_{n}(D), \quad U_{\infty} \equiv \bar{u}(D)$.

Since $u_{n}(x)$ and $\bar{u}(x)$ have a continuous inverse, the sets $U_{n}$ are simply connected and bounded and their boundaries anproach the boundary of $U_{x}$. Thus for each pair of sets $\left\{U_{+}, U_{-}\right\}$such that $U_{-} \subset U_{\infty} \subset U_{+}$there is an $N$ such that $U_{-} \subset U_{n}$ $\subset U_{+}$, if $n>N$. Substitution of $x=x_{n}(u)$ in (B.1) yields

$\sup _{u \in U_{n}}\left\|u-\bar{u}\left(u_{n}(u)\right)\right\| \leqq G_{D} \delta^{n}$.

Since $\bar{x}(u)$ is analytic on $U_{+}$there is a constant $R$ such that

$$
\sup _{u_{1}, u_{2} \in U}\left\|\bar{x}\left(u_{1}\right)-\bar{x}\left(u_{2}\right)\right\| \leqq R\left\|u_{1}-u_{2}\right\| .
$$

Finally combination of (B.7) and (B.8) results in

$$
\sup _{u \in U}\left\|\bar{x}(u)-x_{n}(u)\right\| \leqq R C_{D} \delta^{n} .
$$

It remains to show that for each $u$ there is a $D$ and a $U$ such that $u \in U_{-}$. To find such a $U$ choose $D$ so large that $u$ is interior point of $U_{x}$. Then $U$ can always be chosen in such a way that it contains $u$. QED

\section{Appendix C}

A constant $q$ is calculated such that (3.13) holds for the Hénon mapping (2.1). The result is

$q=2 b^{1 / 2}\left(b-c^{2}\right)^{1 / 2}\left(b^{1 / 2}-|c|\right)^{-1}$.

Choose the matrix $S$ in (3.6) as

$S=\left(\begin{array}{cc}1 & -\lambda^{*} \\ -1 & \lambda\end{array}\right)\left(2\left(b-c^{2}\right)\right)^{-1 / 2}$.

then

$A=\left(\begin{array}{cc}1 & -c \\ -c & b\end{array}\right)\left(b-c^{2}\right)^{-1}$

Eq. (2.1) yields

$(Q(x), A Q(x))=4 A_{11} x_{1}^{4}$.

Since the matrix elements $A_{11}$ and $A_{22}$ are positive, it holds the inequality

$x_{1}^{2} \leqq A_{11}^{-1}\left(A_{11} x_{1}^{2}+A_{22} x_{2}^{2}\right)=A_{11}^{-1}(x, D A x)$,

where $D A=\operatorname{diag}\left(A_{11}, A_{22}\right)$. If $p$ is the smallest constant such that

$(x, D A x) \leqq p(x, A x)$,

then combination of (C.4) and (C.5) shows that (3.13) holds if

$q^{2}=4 p^{2} / A_{11}$. 
Relation (C.6) holds if $(x,(D a-p A) x) \leqq 0$ for all $x$, equivalently if $D A-p A$ has no positive eigenvalues. This condition is satisfied if

$\operatorname{Tr}(D A-p a) \leqq 0, \quad \operatorname{det}(D A-p A) \geqq 0$.

Evaluation of these inequalities (cf. (C.3)) yields

$(1+b)(1-p) \leqq 0, \quad b(1-p)^{2}-c^{2} p^{2} \geqq 0$.

From the first inequality one obtains $p \geqq 1$. The second inequality then gives

$b^{1 / 2}(p-1) \geqq|c| p$.

The smallest value of $p$ satisfying this last inequality is

$p=b^{1 / 2} /\left(b^{1 / 2}-|c|\right)$.

(C.1) then follows readily with (C.7).

\section{References}

[1] V.I. Arnol'd, Geometrical Methods in the Theory of Ordinary Differential Equations (Springer, New York, 1983).

[2] M. Hénon, Comm. Math. Phys. 50 (1976) 69.

[3] J. Guckenheimer and P.J. Holmes, Nonlinear Oscillations, Dynamical Systems, and Bifurcation of Vector Fields (Springer, New York, 1983).

[4] C. Grebogi, S.W. McDonald, E. Ott and J.A. Yorke, Phys. Lett. A 99 (1983) 415.

[5] S.W. McDonald, C. Grebogi, E. Ott and J.A. Yorke, Physica 17D (1985) 125.

[6] R.H.G. Helleman, in: Long-time Prediction in Dynamics, C.W. Horton, L.E. Reichl and A.G. Szebehely, eds. (Wiley, New York, 1983), p. 95.

[7] R.H.G. Helleman, Self-generated behavior in nonlinear mechanics, in reprint selection: Universality in Chaos, $P$. Cvitanović, ed. (Adam Hilger, Bristol, 1983).

[8] Z. Nitecki, Differentiable Dynamics (MIT Press, Cambridge, MA, 1971)

[9] P.J. Holmes and D.W. Whitley, Phil. Trans. Roy. Soc. A 311 (1984) 43.

[10] D.L. Hitzl and F. Zele, Physica 14D (1985) 305.

[11] S.E. Newhouse, in: Chaotic Behavior of Deterministic Systems, G. Iooss, R.H.G. Helleman and R. Stora, eds. (North-Holland, Amsterdam, 1983), p. 381.

[12] T.P. Valkering, Physica 18D (1986) 483.

[13] R. van Damme and T.P. Valkering, J. Phys. A, to appear. 